\title{
The Study of Reactive Intermediates in Condensed Phases.
}

\author{
Barry K. Carpenter, ${ }^{*} \dagger$ Jeremy N. Harvey ${ }^{\ddagger}$, Andrew J. Orr-Ewing \\ ${ }^{\dagger}$ School of Chemistry, Cardiff University, Park Place, Cardiff, CF10 3AT, UK \\ *Department of Chemistry, KU Leuven, Celestijnen Laan 200F, B-3001 Heverlee, Belgium \\ ISchool of Chemistry, University of Bristol, Cantock's Close, Bristol BS8 1TS, UK
}

\begin{abstract}
Novel experimental techniques and computational methods have provided new insight into the behavior of reactive intermediates in solution. The results of these studies show that some of the earlier ideas about how reactive intermediates ought to behave in solution were incomplete or even incorrect. This perspective summarizes what the new experimental and computational methods are, and draws attention to the shortcomings that their application has brought to light in previous models. Key areas needing further research are highlighted.
\end{abstract}

\section{Introduction.}

\subsection{What is a Reactive Intermediate?}

For most of the history of Physical Organic chemistry, reactive intermediates have constituted important subjects of investigation. ${ }^{1}$ They have had their own taxonomy, with classes such as carbocations, carbenes, carbanions, and free radicals being familiar to all organic chemists. Until recently, they were typically not directly observable, which is what led them to be classified as "reactive." An unobservable intermediate is kinetically irrelevant unless it occurs at a branch-point in a mechanism. ${ }^{2,3}$ As a consequence, much of the historical discussion of reactive intermediates has concerned their invocation as the explanations for multiple product formation in reactions.

\subsection{Commonly Accepted Behavior for Reactive Inter-} mediates.

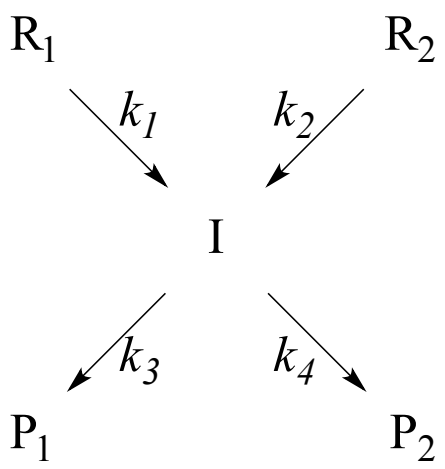

Scheme 1. A typical representation of a reactive intermediate, I, two reactants, $R_{1}$ and $R_{2}$, and two products, $P_{1}$ and $P_{2}$.

Two key assumptions about the behavior of reactive intermediates have played significant roles in their study in the past. These assumptions are linked to the intermediates' association with mechanistic branch points. The first is that a reactive intermediate should proceed on to products in a way that is independent of its mode of generation. Hence, the product ratio from a putative intermediate should be the same no mat- ter how one generates it, provided the other parameters of the reaction (solvent, concentration, temperature, etc.) can be kept constant.

The second assumption is that the equilibrium geometry of the intermediate, particularly its symmetry, should be reflected in the product ratio. A classic example of the use of this second principle is the expectation that an achiral intermediate in an achiral environment should give achiral or racemic products under all circumstances.

These assumptions may seem to be self-evidently correct, because a kinetic analysis of Scheme 1 gives $\left[\mathbf{P}_{1}\right] /\left[\mathbf{P}_{2}\right]=k_{3} / k_{4}$. The product ratio apparently does not depend on $k_{1}$ or $k_{2}$. Hence one could generate the intermediate purely from $\mathbf{R}_{\mathbf{1}}\left(k_{2}\right.$ $=0$ ) or purely from $\mathbf{R}_{2}\left(k_{1}=0\right)$, and the product ratio would be unaffected, supporting the first assumption. If there were a symmetry element in this Scheme, such that $\mathbf{R}_{\mathbf{1}}$ and $\mathbf{R}_{\mathbf{2}}$ were enantiomers, $\mathbf{I}$ were meso (achiral), and $\mathbf{P}_{\mathbf{1}}$ and $\mathbf{P}_{\mathbf{2}}$ were enantiomers, then the transition states between $\mathbf{I}$ and the two products would be enantiomeric, apparently making $k_{3}=k_{4}$, and thereby supporting the second assumption.

However, it has typically not been made explicit in the discussion and application of these principles that the kinetic analysis on which they depend presupposes the validity of the socalled statistical approximation, ${ }^{4}$ which posits that intramolecular vibrational-energy redistribution (IVR) will always be much faster than any chemical event. In addition, it has typically been assumed that solvent reorganization would be sufficiently fast that any symmetry properties of the solute would be expressed in solution-phase reactions.

Under conditions where the statistical approximation is valid an intermediate can carry no dynamical "memory" of its origins, and so its behavior should be deducible simply from the potential energy surface (PES) for the reaction. The only note of caution that one might add to this last claim is that there could still be chemical activation phenomena to be considered for a proper description of the mechanism; in other words an intermediate that was formed in an exothermic step might carry excess energy in vibrations, rotations and translation, which would need to be accounted for in the mechanism. Such effects could complicate the application of the first principle, but should have no influence on the second one. 


\subsection{Intermediates Behaving Badly}

The Physical Organic Chemistry literature carries numerous reports of reactive intermediates apparently not adhering to the code of conduct elucidated in the previous section. ${ }^{5}$ Such reports were especially prevalent in the years following the publication of the Woodward-Hoffmann rules for pericyclic reactions, ${ }^{6}$ as experimentalists set about the task of assessing whether nominally pericyclic processes were actually singlestep, concerted reactions, or whether they occurred in a stepwise fashion, involving intermediates. ${ }^{7-13}$ Attempts to test for intermediates by the symmetry criterion alluded to in Section 1.2 frequently led to ambiguous results. For many reactions, there was evidence of branching to more than one product - an outcome seemingly inconsistent with a single-step mechanism - but product ratios were often not consistent with the anticipated symmetries of the putative intermediates. Included were cases in which reactions occurring via supposedly achiral intermediates afforded optically active products. ${ }^{5,10}$

Such outcomes were typically explained by mixed mechanisms; suitable combinations of concerted and stepwise reactions (with the intermediates in the stepwise components being presumed to be well behaved in their branching ratios), or possibly combinations of "allowed" and "forbidden" concerted reactions, could generally be found that would match the observed results. ${ }^{5}$ Nominally pericyclic reactions have not been the only ones for which it has been necessary to add previously unsuspected pathways in order to explain an observed product ratio. ${ }^{14,15}$

\subsection{Changes in the Code of Behavior}

In the last two decades, or so, a number of developments in chemistry have caused the key principles described in Section 1.2 to be brought into question. The first is that ultrafast spectroscopies of various kinds have allowed a number of reactive intermediates to be detected directly and, in some cases, have additionally allowed an unambiguous tracking of the energy flow during the formation and reaction of these intermediates. This work is summarized in sections 3 and 4 below.

The second important development has been the appearance of the hardware and software which, together, have allowed computation of credible, multidimensional PESs for organic reactions (or, at least, portions of them). These surfaces have often proven not to be consistent with the ad hoc mixedmechanism explanations described in Section 1.3. ${ }^{16,17}$ In addition, the calculations have shown the common occurrence of phenomena not previously considered in the mechanistic principles summarized above. Prominent among such phenomena are reaction-path bifurcations, which constitute branch-points in a mechanism that are not associated with the local minima on the PES normally considered to be prerequisites for the existence of intermediates. ${ }^{18,19}$ Except under special circumstances described below, kinetic models that depend on the validity of the statistical approximation - notably simple versions of Transition State Theory (TST) - are incapable of making predictions about what the branching ratio will be at these bifurcations. A schematic representation of a bifurcating reaction path is shown in Fig. 1.

Even when the branching of reaction paths does occur at a local PES minimum, classical molecular dynamics (MD) simulations have shown that one cannot rely on the validity of the statistical approximation for gas-phase reactions. ${ }^{15,16,18-36}$ The

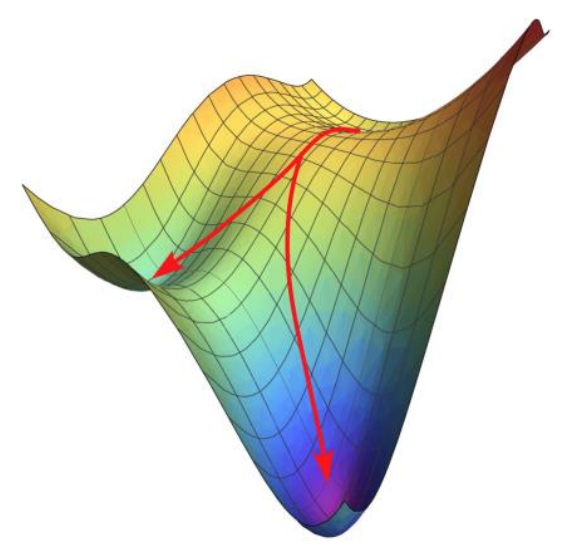

Figure 1. Schematic depiction of a PES with a bifurcating reaction path.

physical mechanisms that lead to IVR in an isolated molecule are not very different from those that lead to chemical transformation, and so, with hindsight, perhaps it was questionable whether one should have assumed that the time required for the former could be very much smaller than for the latter, as the statistical approximation requires.

But this Perspective concerns reactive intermediates in condensed phases. How, if at all, does the presence of a dense surrounding medium (typically a solvent) change the behavior of transient intermediates? It is probably fair to claim that the prevailing view in the organic chemistry community is that a solvent provides a highly efficient energy sink, and so the chemical activation phenomena described above need not be considered. In the gas phase, it has long been recognized that this is not always true: in the low-pressure limit of gas phase reaction kinetics, thermalizing collisions with other gas molecules can occur much slower than unimolecular bond-breaking and making. This was first suggested by Lindemann, in a comment ${ }^{37}$ where he hypothesized that incomplete thermalization could most easily be detected in gas phase reactions at low pressure. However, he went on to state that:

"In liquids of course no such effect could occur, since in these the Maxwell distribution must be rapidly re-established by collisions with the solvent molecules."

If the solvent were perfectly efficient in removing excess energy from a reacting solute, then the reaction would always follow exactly the steepest-descent path on the PES - in other words the Intrinsic Reaction Coordinate (IRC) - and one would even be able to make predictions about branching ratios for reactions with bifurcating paths to products.

The second prevailing view is that solvents can influence the behavior of reactive intermediates by effects that change the shape of the PES. These will usually be couched in polarity terms (with hydrogen bonding here being included as a special case of a polar effect). There is common discussion in the organic chemistry literature of the stabilization of ionic intermediates by polar solvents, and consequent effects of the solvent on the overall reaction mechanism. ${ }^{38}$ In such discussions there is usually the implicit (sometimes explicit) assumption that the solvent can respond instantaneously to changes in solute polarity. ${ }^{39}$ 
However, as this Perspective will seek to illustrate, there are reasons to believe that these common assumptions about the effects of solvents are incomplete and, in some cases, simply wrong. On the energy front, for example, it is notable that Baldwin ${ }^{40}$ wrote the following in 1977:

"One may anticipate that hot-molecule chemistry in solution will receive fresh attention; the conveniently simplifying generalization that collisional deactivation in solution always preempts thermal reactions of vibrational excited molecules can no longer be credited."

He made that statement following his group's experimental verification of an earlier proposal by Brauman ${ }^{41}$ and by Flowers and Frey ${ }^{42}$ that an unusual rearrangement accompanying the ring opening of a strained hydrocarbon in solution could be the result of incomplete quenching by the solvent of the excess energy in the nascent product. However, it appears that Baldwin's anticipation of a change in view about solvent effects on reaction energetics has taken about four decades to be realized, and even now the new view is far from universally accepted.

Even if one does accept the idea that intermolecular energy transfer from solute to solvent may not be perfectly efficient, there is a separate question about the influence of the medium on intramolecular energy transfer, i.e. IVR, within the solute. Is there reason to think that fluctuations (pseudo-collisions or rapid changes in local polarity, for example) imposed by the solvent on the solute will lead to enhanced IVR and hence closer adherence to the behavior anticipated by TST? There is not a complete answer to that question at hand yet; in fact there is not even complete agreement among the authors of this Perspective about the likely answer, but it seems to be a question worth asking.

Finally, one may ask whether existing models used in the simulation of solvent effects on chemical reactions are adequate to capture all of the important phenomena in reactive intermediate behavior. Again, there is preliminary evidence that the answer may be "no," but a lot more work needs to be done.

The remainder of this Perspective will provide brief summaries of what the current models for solvent effects on chemical reactions are, what the newly developing experimental techniques can offer, and then a summary of the evidence that there can be important effects in condensed-phase reactive intermediate chemistry which conventional views of solvent effects are not fully capturing.

\section{Common Models for Solvent Effects on Or- ganic Reactions}

In experimental Physical Organic Chemistry, solvent effects have typically been treated by various kinds of linear free energy relationships, all of which focus on the polarity of the medium. ${ }^{43}$ In special cases, such as in the discussion of caged radical pairs, there may additionally be consideration given to the viscosity of the medium as a controlling feature, ${ }^{44}$ and properties such as the internal pressure of the solvent have also occasionally been considered, ${ }^{45}$ but these are much less common than the discussions of solvent polarity.

In computational chemistry, treatment of solvents broadly falls into two classes of approach - implicit and explicit. In the implicit models the solvent is typically represented by some dielectric continuum, which polarizes in response to the solute polarity. In explicit models one has representations of individual solvent molecules, often constrained in a periodic- boundary box, which may then be treated by Monte Carlo or molecular dynamics techniques.

Early versions of the implicit solvent models were all based on some version of linear-response theory, ${ }^{46}$ and indeed that remains the foundation of more modern versions. ${ }^{47}$ The early versions also treated solvation as an equilibrium phenomenon - i.e. assuming that the solvent could always respond instantaneously to changes in the solute. However, as nicely summarized in a review by Cramer and Truhlar, ${ }^{48}$ there is now recognition that in the treatment of reaction dynamics, such models of solvation may be inadequate. There may be significant, specific interactions of the solute with atoms of the solvent in the first solvation shell occurring during the reaction. Attempts to reproduce such effects, while retaining the spirit of an implicit solvation model, have typically involved inclusion of microscopic viscosity terms for the solvent in the first solvation shell. ${ }^{48}$

In principle, explicit solvation models could more readily handle non-equilibrium solvation effects in reaction dynamics than implicit solvation models do. However, as usually implemented, the explicit models are not really much better. The reason is that the typical approach is to compute a potential of mean force (PMF) or free energy profile for the reaction in the solvent. ${ }^{4,50}$ In this procedure one picks some reaction coordinate for the solute and then computes thermally equilibrated solvation energies for steps along that coordinate in order to find the free energy maximum. By so doing, one is giving primacy to the solute in determining the reaction coordinate, with the solvent playing a secondary, perturbative role. This is likely to be problematic for the rather common situation in which a reactive intermediate is formed in the ratedetermining step of a reaction and then goes on to give products in a set of parallel, low-barrier reactions. Under such circumstances it may not be possible for the solvent to equilibrate fully around the transition structures for the productforming steps. If that is indeed the case, then calculations of free energy barriers for the product forming steps, no matter how accurately the underlying thermodynamics may be modeled, will be unlikely to predict correct branching ratios.

Changes in polarity of the solute during a reaction have formed the focus of most attempts to model solvent effects on kinetics. Changes in shape of the solute have received much less attention, but for reasons outlined below, may be important to consider. In the few studies that have considered changes in shape of the solute, the conclusion seems to have been that linear response theories are unlikely to be adequate. ${ }^{51,52}$ This is, at least in part, because the non-bonded terms that represent steric interactions are highly nonlinear, as exemplified by the Lennard-Jones potential with its inverse $6^{\text {th }}$ and $12^{\text {th }}$ power distance terms for attraction and repulsion, respectively. A $R^{-12}$ repulsion can go from being all but negligible to completely dominant in a small range of $R$, and hence on small time scales during a reaction.

In order to see why equilibrium solvation models are unlikely to be adequate for the description of condensed-phase dynamics of reactive intermediates, it will be useful to summarize what experimental methods are available now for studying such reactions, and what information has come out of them about time scales for energy flow and solvent reorganization. 


\section{Experimental techniques for studying solu- tion phase reactive intermediates}

Short lifetimes and low steady-state concentrations make reactive intermediates difficult to observe in reaction mixtures. The rapid loss of these species in solution might result from reactive removal, isomerization, fragmentation, recombination of radical pairs, or quenching of excited states. Nevertheless, various time-resolved spectroscopic techniques can capture in situ signatures of these elusive, but mechanistically important, intermediates and determine their rates of production and decay. Reactive intermediates can also be trapped for extended study by adsorption to a cold surface or by rapid condensation into a cryogenically cooled and chemically inert matrix. Recent breakthroughs in single-molecule imaging based on atomic force microscopy (AFM) and scanning tunneling microscopy (STM) are enabling the mapping of structures of reactive intermediates frozen on solid surfaces under ultra-high vacuum; for example, Pavlicek et al. recently reported AFM observation of arynes produced by elimination of two iodine atoms from a polycyclic aromatic ortho-diiodoarene molecule. ${ }^{53}$ Rare-gas, ${ }^{54,55}$ and more recently para-hydrogen, ${ }^{56}$ matrices have been extensively used in combination with infra-red absorption spectroscopy to characterize vibrational modes of the intermediates, and the development of superfluid helium nanodroplets as inert hosts now affords rotationally resolved spectra of exotic species. ${ }^{57,58}$ However, these experimental studies of isolated and trapped molecules do not generally reveal the chemical lifetimes of the intermediates or their reaction dynamics.

\subsection{Time-resolved studies of reaction intermediates}

Time-resolved spectroscopy can provide both chemical characterization and kinetic information on reactive intermediates, and can probe these species in situ without trapping or isolation steps. Flash photolysis of a precursor is a wellestablished method to generate radicals by homolytic bond cleavage, and reactions can also be initiated by the similar approach of photoexcitation to a reactive electronically excited state. These photo-initiation strategies form the basis of our discussion of experimental probes of reactive intermediates. The magnetic moments of radical intermediates make them accessible to study by electron paramagnetic resonance (EPR) on microsecond or longer timescales, ${ }^{59,60}$ and measurements of magnetic field effects are helping to unravel complex photochemical pathways involving radical pairs, such as those hypothesized to allow birds to navigate using the Earth's magnetic field. ${ }^{61}$ The reacting species and their solvent environments will be equilibrated on these timescales because of thermalizing interactions with the solvent. However, time resolution in the femtosecond to picosecond regime is required if the non-equilibrium dynamics of reaction pathways are to be fully characterized, because these short intervals correspond to the timescales of bond cleavage, electron transfer, structural isomerization, excited state relaxation, and solvent reorganization to accommodate a reactive event. There is also valuable mechanistic information in the disposal of excess energy of exothermic processes into motions of the reactive intermediates, and its subsequent flow into the solvent bath. ${ }^{25,62}$ To access "ultrafast" time intervals, methods based on femtosecond optical (UV, visible and IR) or X-ray pulses are necessary and new technologies are rapidly expanding our scope to study chemical reactions under non-equilibrium conditions.

\subsubsection{Transient absorption spectroscopy}

An ultrafast transient absorption study of a chemical reaction is conceptually simple. ${ }^{63}$ An ultraviolet or visible (actinic) "pump" laser pulse of duration shorter than $100 \mathrm{fs}$ initiates reaction by inducing bond cleavage or excitation to a reactive state. A second pulse of similar duration probes the subsequent chemistry by absorption of infrared or UV/visible wavelengths. The pump and probe pulses are synchronized by generating them from the same output pulse of an amplified ultrafast laser, and the probe is then time-delayed with respect to the pump pulse using an optical delay stage of controlled and variable length (an additional $3 \mathrm{~mm}$ path introduces a delay of $10 \mathrm{ps}$ ). Selection of a set of delay times maps out a kinetic trace of the growth and decay of absorption bands associated with intermediate species. The laser pulses span a range of wavelengths as a direct consequence of their short durations, and the wavelength coverage can be further extended by nonlinear optical processes such as white-light continuum generation across the near-IR, visible and near-UV regions. ${ }^{64}$ For example, a sub-100 fs duration mid-IR pulse centered at a wavenumber of $1700 \mathrm{~cm}^{-1}$ might span the entire range from $1500-1900 \mathrm{~cm}^{-1}$ with sufficient intensity for spectroscopic measurements. ${ }^{65}$ A spectrometer equipped with a grating and a multi-element array detector can simultaneously measure the transmission of all these wavenumber (or wavelength) components by a reaction mixture, allowing rapid identification of any intermediate species generated by the pump laser pulse. The limiting time resolution of the measurements is determined by the cross correlation of the pump and probe laser pulses, and is commonly referred to as the instrument response function (IRF). With pulse compression and careful optical design to prevent stretching of ultrashort pulses, IRFs below 50 fs can be achieved.

Transient vibrational absorption (TVA) spectroscopy uses ultrafast IR probe pulses to observe reactants, intermediates and products after initiation of reaction by an actinic pulse. An example is presented in Fig. 2 in which TVA spectra of a $U V$-excited $\alpha$-pyrone solution illustrate heterocyclic ringopening to a ketene photoproduct. ${ }^{66}$ In the usual representation of TVA spectra, consumption of reactants appears as negative going, or "bleach" features, whereas bands deriving from intermediates and products are positive. ${ }^{65}$ Stable products give absorption bands that show steady growth and remain over extended times, but the bands of reactive intermediates grow and decline as the intermediates are formed and lost. Vibrational cooling of initially hot molecules manifests as a shift of bands to higher wavenumber (because of vibrational anharmonicity), as seen in Fig. 2(b), ${ }^{67,68}$ whereas solvent response towards equilibrium confers shifts to lower wavenumber that are most pronounced in hydrogen bonded systems. Absorption bands can readily be assigned to reactive intermediates by applying modest solvent shifts (generally to lower wavenumber) to known gas-phase or matrix-isolation spectra, or by comparison with electronic structure calculations of vibrational mode frequencies. ${ }^{69}$

The bands observed in time-resolved UV/visible spectra are transient electronic absorption (TEA) features that tend to be broader and more overlapped than mid-IR TVA bands, and to exhibit larger solvent shifts, making decomposition into spectral components and assignment more difficult. ${ }^{70}$ However, new bands can contribute to the spectra in addition to those 

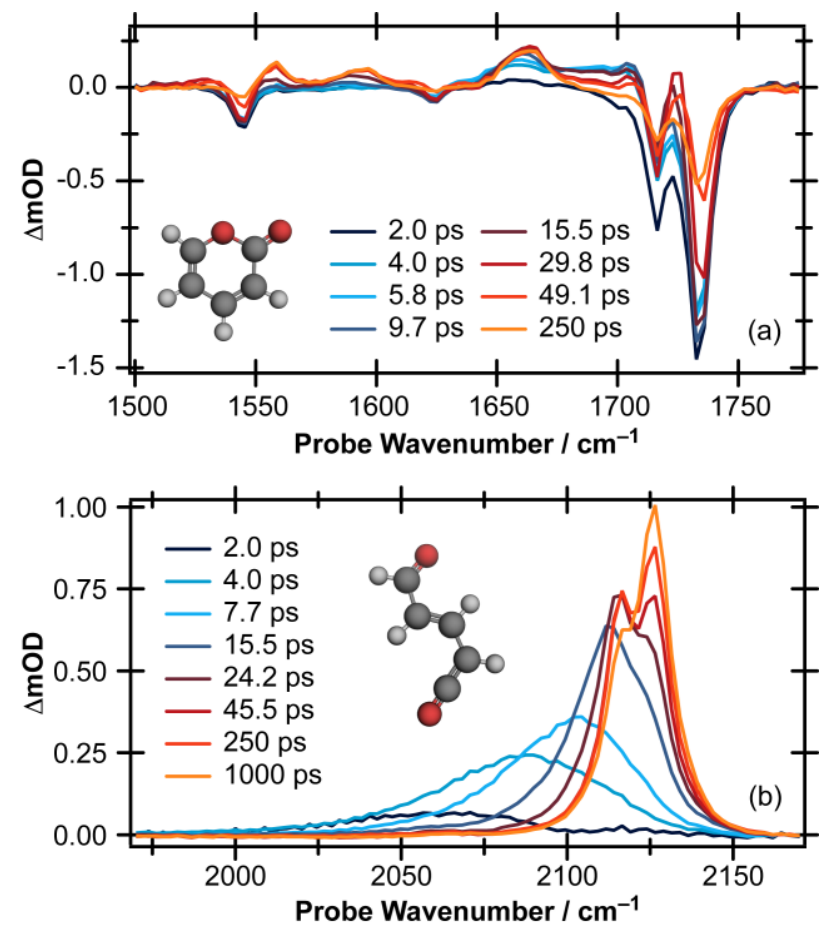

Figure 2. TVA spectra of a $30 \mathrm{mM}$ solution of $\alpha$-pyrone in acetonitrile following excitation with $310-\mathrm{nm}$ UV light. The inset color keys identify spectra obtained at different pump-to-probe laser time delays. (a) Bleach features in the carbonyl stretching region show initial depletion and subsequent recovery (reduction in the depth of the bleach) of the parent molecule. (b) Transient absorption bands in the ketene stretching region show formation of ring-opened photoproducts. The narrowing and shifting of the band to higher wavenumber indicate initial formation of vibrationally hot products (reflecting the absorbed photon energy and the structural change on ring-opening) that cool over timescales of tens of ps. Data were provided by D. Murdock (University of Bristol).

between electronic states of the solute molecules. These additional spectral features can be advantageous; for example, bands corresponding to charge-transfer from/to the solvent, or distinct spectral signatures of solvent-complexed radicals help to characterize the solvation environment of a reactive species and its influence on reaction rates. ${ }^{71-73}$ The time dependence of IR or UV/vis bands provides further evidence to support proposed assignments.

A recent study of fluorine atom reactions in $d$-acetonitrile illustrates application of TVA and TEA methods to chemical dynamics in solution. ${ }^{74} \mathrm{XeF}_{2}$ dissolved in $d$-acetonitrile served as a precursor to $\mathrm{F}$ atoms, which were liberated by UV excitation using a $267-\mathrm{nm}, 50 \mathrm{fs}$ duration laser pulse. TEA spectroscopy of the resulting solution probed the $\mathrm{XeF}$ photoproduct via its $\mathrm{B}-\mathrm{X}$ absorption band in the near UV. The close proximity of the partner F-atom broadened this band to longer wavelength, and the decay of this shoulder indicated loss of the F atoms with a time constant of $4 \mathrm{ps}^{75}$ TVA measurements revealed the growth of product DF absorption bands in the $2350-2650 \mathrm{~cm}^{-1}$ region, with spectral shifts indicating cooling of initially vibrationally excited DF and a slower response of the surrounding solvent to the changing chemical composition of the solute as well as the release of $\sim 40$ $\mathrm{kcal} / \mathrm{mol}$ of energy from the reaction. ${ }^{74}$ These TEA and TVA methods are also finding wide application in photochemical studies, for example of spin-crossover dynamics in transition metal complexes following excitation of a metal-to-ligand charge-transfer (MLCT) band, ${ }^{76}$ or excited state reaction and relaxation pathways in solvated nucleobases, ${ }^{77}$ nucleobase pairs, ${ }^{69}$ and single and double strands of DNA. ${ }^{78,79}$

\subsubsection{Two-dimensional IR and UV spectroscopy}

Two-dimensional versions of vibrational (2DIR) and electronic (2D visible and UV) spectroscopy provide information about couplings between vibrational or electronic states respectively of molecules on ultrafast timescales. ${ }^{80-84}$ 2DIR has also been used to observe conformational exchange in fluxional molecules such as $\mathrm{Fe}(\mathrm{CO})_{5},{ }^{85}$ or chemical exchange in solution, ${ }^{86}$ processes which are too fast to be followed by NMR. Fig. 3 shows measurements from our laboratory of ligand-site interconversion by pseudo-rotation in $\mathrm{Fe}(\mathrm{CO})_{5}$. These coherent $2 \mathrm{D}$ optical spectroscopies require a sequence of two or more ultrafast pulses, and are most commonly implemented with a 3-pulse sequence (two pump pulses and a probe pulse). If $2 \mathrm{DIR}$ is to be used to study reaction mechanisms, an additional actinic pulse must be introduced to generate reactive intermediates. This transient 2DIR (t-2DIR) approach remains experimentally challenging, with signals that are significantly weaker than normal TVA measurements, and only a few examples have been reported to date. ${ }^{87}$ The hybrid $2 \mathrm{DEV}$ method examines the coupling between electronic and vibrational (i.e., nuclear) motions in molecules, ${ }^{88,89}$ and has the potential to examine reactive intermediates corresponding to photoexcited states.
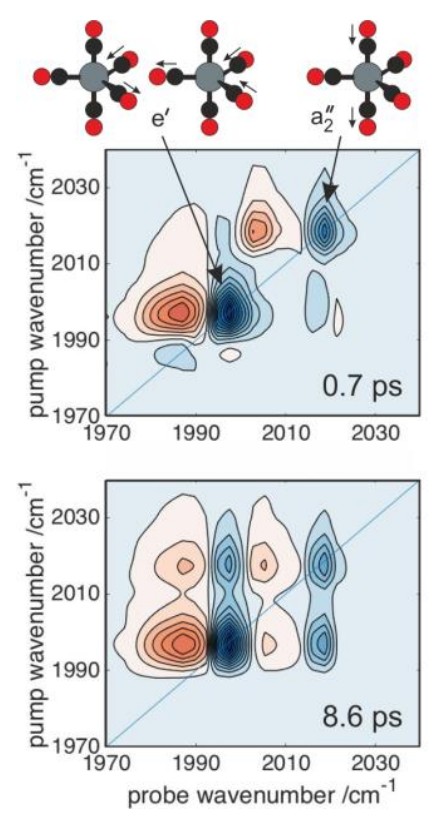

Figure 3. 2DIR spectra of pseudo-rotation dynamics in $\mathrm{Fe}(\mathrm{CO})_{5}$ dissolved in $n$-dodecane. At short time delays $(0.7 \mathrm{ps}$, top panel) between the pump and probe IR pulses, the spectrum is dominated by features on the diagonal corresponding to the degenerate (e') stretching modes of the equatorial CO ligands (at $1999 \mathrm{~cm}^{-1}$ ) and the a2" stretching mode of the axial ligands (at $2022 \mathrm{~cm}^{-1}$ ). After a delay of 8.6 ps (bottom panel) exchange between axial and equatorial sites contributes off-diagonal cross peaks to the 2DIR 
spectrum. Data were provided by H.J.B. Marroux (University of Bristol).

\subsubsection{Time-resolved photoelectron spectroscopy}

With the advent of technology to introduce liquids into high vacuum apparatus in the form of micro-jets, photoelectron spectroscopy has emerged as a viable method to probe solutes in aqueous and other solvent media, ${ }^{90,91}$ and to study photochemical and reactive intermediates in solution. Photoelectron spectroscopy of small clusters in molecular beams also examines solvent effects on reaction intermediates, and control of the number of solvent molecules in the solvation shell is possible using mass spectrometric selection of specific cluster sizes. To observe intermediate species and measure their lifetimes, photoelectron spectroscopy can be performed with ultrafast time resolution, using femtosecond laser pump and probe (ionization) schemes. ${ }^{92}$ Recent examples include study of electron attachment to nucleobases in clusters, ${ }^{93,94}$ and charge-transfer-to-solvent reactions for $\mathrm{I}^{-}$in aqueous solution. ${ }^{95}$

\subsubsection{Time-resolved $X$-ray spectroscopies}

Substantial strides have been taken in recent years to develop spectroscopic probes of reaction intermediates using ultrafast X-ray pulses. ${ }^{96,97}$ These short wavelengths excite core electrons to valence orbitals or the ionization continuum, and are element specific and sensitive to the oxidation state and local chemical environment of the probed atoms. Measurements to date have mostly used synchrotron light sources, with time resolution reaching the picosecond range, although adapted synchrotrons and X-ray free electron lasers (XFELs) access picosecond and femtosecond timescales. ${ }^{98-100} \mathrm{X}$-ray absorption spectroscopy (XAS) measures transitions from deep core (1s, $2 \mathrm{~s}, 2 \mathrm{p}$, etc.) orbitals to higher lying unoccupied orbitals or the ionization continuum. Just above the absorption edge corresponding to ionization of the core electron, XANES (X-ray absorption near edge structure) reveals bond distances and bond angles. To higher energy, EXAFS (extended X-ray absorption fine structure) yields atomic arrangements in the form of radial distribution functions around the probed atom. Variants such as X-ray fluorescence spectroscopy, ${ }^{101} \mathrm{X}$-ray photoelectron spectroscopy and resonant inelastic X-ray scattering $\left(\right.$ RIXS) ${ }^{102}$ have explored processes such as spin-crossover dynamics following photo-induced MLCT. Examples in the literature mostly focus on evolving electronic structure, changes in spin state and structural changes in coordination complexes of transition metals (TMs) and metalloproteins. ${ }^{97}$ Access to synchrotron or XFEL beam time has so far limited the application of these X-ray based methods, but table-top laser sources of extreme ultraviolet (XUV) and X-ray radiation are becoming available for laboratory (rather than national facility) based studies, ${ }^{96}$ and should lead to further exploitation of these powerful techniques. For example, Leone and coworkers recently showed that XUV pulses produced by high harmonic generation of 35-fs pulses from a titanium:sapphire laser could probe core-to-valence transitions in iodine atoms during the 266-nm induced dissociation of the C-I bond in methyl iodide. ${ }^{103}$

\subsubsection{Ultrafast diffraction methods}

Although spectroscopic probes provide incisive information about vibrational frequencies, electronic absorptions, and elemental composition of reactive intermediates, a long-held aspiration is to image the structures and motions of the interme- diates directly by diffraction methods. ${ }^{104}$ Advances have therefore been sought in generating short pulses of electrons or $\mathrm{X}$-rays for time-resolved diffraction studies. Electron pulses have been developed that are capable of imaging dynamical processes on the $\sim 10$ fs timescale, ${ }^{105}$ but these and ultrafast Xray diffraction are only just beginning to be applied to studies of reactive intermediates.

\section{Experimental Data on Solvent Response Timescales}

As outlined in Section 1.4, the timescales for motion of solvent molecules, and for redistribution of their energy, are so short that it may seem appropriate to think of the solvent as responding instantaneously to any chemical change within its midst. Shortcomings to this model are quickly revealed by comparison of the timescales for passage of a reaction through a transition state, energy transfer to the solvent bath, and adjustment of the solvent molecules in response to changes in the chemical identity of the solute.

Restructuring of solvent molecules to accommodate changes to a solute molecule can be examined by a variety of timeresolved spectroscopies. Ultrafast studies of Stokes shifts of fluorescence wavelengths in photoexcited dye molecules show how quickly a solvent responds to a change in solute polarity. ${ }^{106-108}$ Two-dimensional infra-red (2DIR) spectroscopy of solvents such as water, and solutes in a variety of media including ionic liquids, quantify molecular equilibration times from direct observation of ultrafast chemical exchange, vibrational relaxation, and spectral diffusion of lineshapes. ${ }^{81} \mathrm{Te}-$ rahertz spectroscopy can probe the cooperative dynamics of the molecules of a solvent around a solute molecule. ${ }^{109,110}$ The solvent response typically shows more than one timescale, corresponding to fast reorientation of molecules in the first few solvent shells, followed by longer range restructuring. ${ }^{106}$ The fastest "inertial" component may have a time constant shorter than $100 \mathrm{fs}$ in solvents such as water, acetonitrile and methanol, but require a few hundred femtoseconds in chloroform, DMSO or DMF. The slower "diffusive" components extend into the picosecond regime. To understand the effect of the solvent on reaction mechanisms, these timescales must be compared to the $<100 \mathrm{fs}$ passage of a chemical reaction through the region of a transition state, or the coupling of excess vibrational energy of a solute to the solvent bath over picosecond or longer intervals. ${ }^{62}$ Direct measurements of $\mathrm{HCl}$ and $\mathrm{HCN}$ vibrational relaxation rates in chlorinated solvents show exponential time constants as long as a few hundred picoseconds to a few nanoseconds, ${ }^{67,111}$ whereas DF vibrationally cools in d-acetonitrile in $3 \mathrm{ps}^{74}$ The rates of these processes depend sensitively on the molecular nature of the solvent, and the strength of coupling of solute and solvent modes. ${ }^{112}$ Energy transfer to the solvent bath is accelerated by near-resonance of the solute vibrations and solvent motions. It is clear that these dynamical solute-solvent interactions will not be correctly captured in models that treat the solvent as a continuum with a dielectric constant to represent its polarity, and that make assumptions of thermal equilibrium throughout a reaction.

Recent ultrafast infrared spectroscopy measurements of exothermic radical reactions in solution show that reacting solute molecules do not maintain equilibrium with the surrounding solvent. ${ }^{67,74}$ Moreover, the excess energy of reaction is efficiently channeled into vibrational modes of the reaction prod- 
ucts, after which it can take several hundred picoseconds to thermalize in weakly interacting solvents such as chloroform or dichloromethane. Accompanying computer simulations demonstrate the importance of correctly treating the molecularity of the solvent to reproduce experimental observations, and the need to take careful account of the coupling between the solute and solvent degrees of freedom. ${ }^{112-114}$ An example trajectory from one such simulation (see also Section 6) is shown in Fig. 4 for DF from reaction of a fluorine atom with a molecule of the surrounding $\mathrm{CD}_{3} \mathrm{CN}$ solvent.

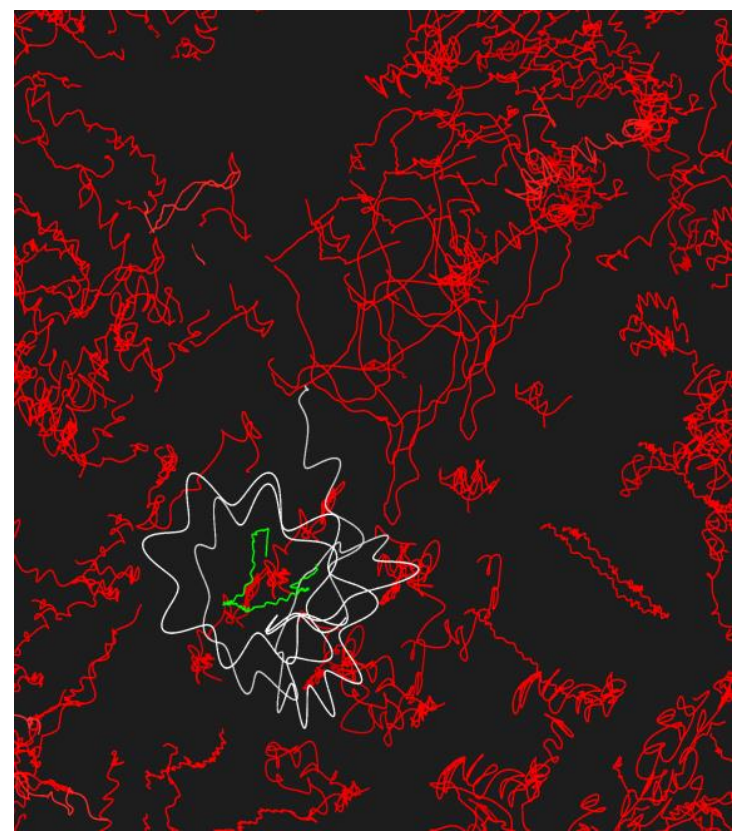

Figure 4. A simulated reaction of an $\mathrm{F}$ atom in d-acetonitrile produces DF that is initially vibrationally excited, and undergoing almost free rotation. The green and white lines respectively trace the motions of the $\mathrm{F}$ and $\mathrm{D}$ atoms of DF, and the star-like pattern indicates both vibrational and rotational motion. The random motions of the surrounding solvent molecules are shown in red. Within $1 \mathrm{ps}$, the DF settles into a hydrogen-bonding interaction with a neighboring solvent molecule, but the vibrational motion requires $\sim 3$ ps to relax to equilibrium. Figure courtesy of D. R. Glowacki (University of Bristol).

\section{Master Equation Treatment of Solution- Phase Reactions}

As described in Section 4, experimental studies of vibrational cooling in solution show that thermalization timescales of the order of a few ps to a few tens of ps are typical. There are even extreme cases such as vibrational relaxation of molecular nitrogen in liquid nitrogen, ${ }^{115}$ where dissipation of the energy of the $\mathrm{v}=1$ state into intermolecular modes has a timescale of $1.5 \mathrm{~s}$. Thermalization can be conceptualized using the isolated binary collision model, ${ }^{116}$ whereby molecules exchange energy with the solvent through isolated "collision" events, occurring every $10^{-13}$ or $10^{-12} \mathrm{~s}$, and which each have the capacity to lead to a change of about $1 \mathrm{kcal} / \mathrm{mol}$ in the vibrational energy of the solute.

The hydroboration reaction of terminal alkenes leads to mixtures of primary and secondary boranes. A $\pi$ complex between the borane and the alkene is a key intermediate in this reaction. This is formed in an exothermic bimolecular step after disso- ciation of the ether solvent from the borane, and is thereby initially formed with excess internal energy, of roughly 11 kcal/mol. ${ }^{117}$ From this common intermediate, reaction can proceed over two different transition states, one leading to the primary borane, and one to the secondary isomer. Because both barriers are very low, reaction proceeds very rapidly, in several ps. Indeed, there is some suggestion that the reaction has partial ballistic or dynamical character, with barrier crossing occurring impulsively immediately without any vibrational energy randomization within the intermediate. ${ }^{117}$ This was suggested to account for the observed ratio of the two isomers, of roughly 9:1, which differs significantly from the 99:1 ratio expected for a thermal reaction, based on accurate quantum chemical calculations that yield a difference in standard free energies for the two competing TSs of $2.5 \mathrm{kcal} / \mathrm{mol}$. However, it is also possible to account for the observed ratio of products by taking into account the incomplete thermalization of the intermediate. ${ }^{118}$ By using RRKM energy-dependent reaction rates for crossing the two TSs, an initial internal energy distribution reflecting the exothermicity of formation of the intermediate, and a binary collision model for thermalization, excellent agreement with experiment was obtained with no need to further assume any role for dynamical behavior (though of course the latter could not be ruled out in this model).

\section{Trajectory Calculations for Solution-Phase Reactions}

As the results on hydroboration summarized in Section 5 illustrate, "hot molecule" effects can play an important role in determining product ratios for reactions in solution, and such effects are seen not only in the reactions of the exotic molecules for which they were first proposed; they apparently need to be considered in the "textbook" reactions of organic chemistry. The hydroboration studies also illustrate that one must be careful in trying to differentiate effects that are due to reactions occurring on a timescale similar to intermolecular energy transfer from solute to solvent from those that are due to reactions occurring on a timescale similar to intramolecular energy transfer within the solute. The former might still be treatable by statistical kinetic models, as the master-equation results showed. The latter, on the other hand, would signal a breakdown of the statistical approximation and would consequently require a different approach. Master-equation models describing energy transfer from one region of the reacting molecule to another can be constructed, though trajectory methods of some type would probably be preferable.

Two questions arise naturally for the latter class of reactions. The first is whether there is any evidence that they even exist. The second is how one would go about doing the calculations. The methodological question will be dealt with first in what follows.

The obvious problem with conducting trajectory calculations on condensed-phase reactions is the size of the task. One will inevitably use direct-dynamics techniques - i.e. computing the potential energy and its derivatives as needed for each step of a trajectory rather than attempting to calculate a full PES ahead of time - but that can be a daunting challenge. A typical calculation on an organic system might involve a solute and a few hundred solvent molecules in a box with a periodic boundary representation. If there were 5000 atoms in total and if one wanted to run a 1 ps trajectory with 0.1 fs time steps then $5 \times 10^{7}$ potential energy calculations and $1.5 \times 10^{8}$ first de- 
rivatives would need to be calculated. And the result of all that is only a single trajectory. A typical simulation might require hundreds to thousands of trajectories in order to sample initial conditions in a meaningful way. It is apparent that one needs to be able to complete each energy and derivative calculation very rapidly for the task to be achievable in a reasonable time. In the explicit-solvent PMF simulations mentioned in Section 2, one can reduce the size of the calculation by using models of solvent molecules which mirror size, dipole moment, and perhaps polarizability and hydrogen bonding capability of the real thing but which do not have explicit representations of every atom. However, this approach is unlikely to be successful if one wishes to simulate properly the influence of the solvent on inter- and intramolecular energy flows. One can bring to bear very substantial computing power, notably these days through the use of Graphical Processing Units, ${ }^{119}$ but even then the necessary calculations cannot yet be done at the levels of electronic-structure theory likely to give the most reliable results for the bonding changes during the reaction under study. At present, then, the only feasible option is to use a relatively low-level method for the solvent - typically some semi-empirical quantum mechanics (QM) method or molecular mechanics (MM) - and then a higher-level method for the solute (and perhaps the nearest few solvent molecules). These might be various versions of so-called QM/MM methods, such as ONIOM, ${ }^{120}$ or they might be purely MM, with the force-field for the solute being modified in some way that permits it to treat bond breaking and formation. Among the latter class, methods that use an Empirical Valence Bond (EVB) approach to generate a reactive force-field have shown promise. $^{112,121,122}$ Finally, it might in special circumstances be possible to model the reaction in a "microsolvation" model in which only a first shell of solvent molecules is included. In such cases the whole solvent-solute complex might be small enough to be treated at a reasonably high level of theory (typically some kind of density functional theory (DFT)).

Let us turn now to the results of such calculations. Two studies from Singleton's group suggest that there can be circumstances in which IVR within a reactive intermediate is incomplete prior to its progress on to products, even in solution. The first study involved ozonolyses of vinyl ethers with alkane side-chains of varying length. ${ }^{31}$ The results have been reviewed in detail elsewhere, ${ }^{123}$ and so will be summarized only briefly here. In short, Singleton's group measured product ratios for the ozonolysis of compounds of the class RO$\mathrm{CH}=\mathrm{CH}_{2}$ in $\mathrm{CD}_{3} \mathrm{OD}$ solution. The two products of interest arise by alternative cleavage pathways from a common intermediate - the primary ozonide generated by 1,3-dipolar addition of ozone to the vinyl ether. The transitions states for the competing cleavages are calculated at the G4 level to be 29 and $35 \mathrm{kcal} / \mathrm{mol}$ below that for the dipolar addition, and so this is one of those cases where the excess energy in the primary ozonide must be treated properly for the branching ratio to be calculated correctly. The next question is whether statistical models can handle that task. Singleton et al. found that they could not. They measured the product ratio as a function of the length of the alkyl side chain, $\mathrm{R}$, and found that the variation was not consistent with models that assumed the excess energy in the intermediate to be fully distributed among all vibrational modes of the primary ozonide prior to the cleavage reactions occurring. They simulated their reaction by an ONIOM model, and found that they could get a good match to the experimental results. From the combined experimental results and simulations, they found that the energy flow within the alkyl side-chain could be reasonably well represented by a first-order process having a rate coefficient of $\sim 4 \times 10^{11} \mathrm{~s}^{-1}$.

In their second study, ${ }^{124}$ Singleton's group again combined theory and experiment to model a solution-phase reaction. The reactant in this case was an aryldiazonium cation, which was undergoing nucleophilic substitution of the nitrogen by water. They measured ${ }^{13} \mathrm{C}$ kinetic isotopes for the reaction, and found that they were not readily explicable by either of the dissociative $\left(\mathrm{S}_{\mathrm{N}} 1\right)$ or associative $\left(\mathrm{S}_{\mathrm{N}} 2 \mathrm{Ar}\right)$ mechanisms that conventional organic chemistry would consider viable. However, when they used a microsolvation model for the reaction, with 10 water molecules being included in DFT directdynamics simulations, they found that the reaction appeared to be on a borderline between the two mechanisms. Further, it appeared that solvent motions played important roles in the reaction coordinate for the overall transformation. Such a finding perhaps serves as a further cautionary note about the use of PMF approaches for treating solvent effects, since they are intrinsically incapable of incorporating solvent motion into the reaction coordinate.

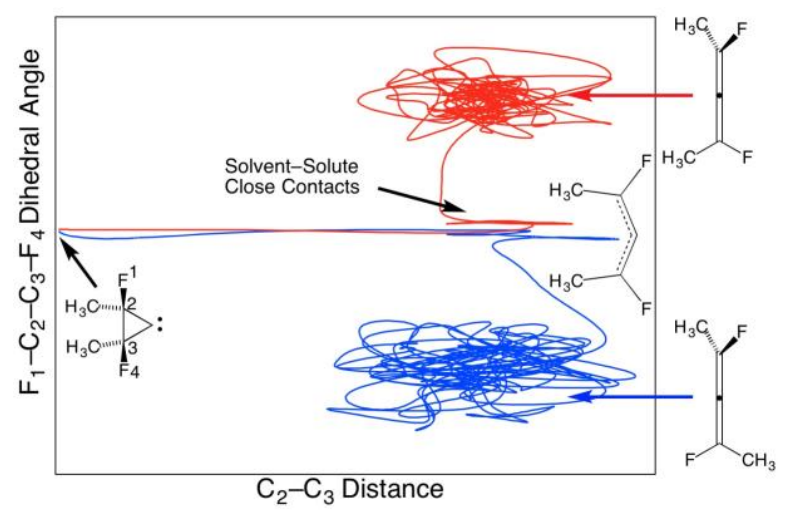

Figure 5. Projections of two trajectories from the ring opening of a cyclopropanylidene in solution. Close contacts between solute and solvent occur as a result of both the change in shape of the reactant and the reaction exothermicity. These close contacts cause changes in direction of the trajectories, and can influence which enantiomer of the allene product is formed when the solvent is chiral and non-racemic.

A recent simulation of a reaction with a bifurcating reaction path has highlighted other solvent effects that cannot reasonably be approximated by equilibrium solvation models. ${ }^{125}$ In this study the ring opening of a substituted cyclopropanylidene (generated from a diazo precursor) was simulated in three different organic solvents. The substituents were selected in such a way that the reactant would be meso, i.e. achiral, whereas the product allenes would be chiral. Each branch of the reaction path bifurcation led to one enantiomer of the product. The simulation was carried out by the EVB-MM method summarized above. The results showed clear evidence of strong, non-equilibrium solvent-solute interactions. These occurred as a result of the substantial change in shape of the solute - with the three carbons of the cyclopropyl ring opening to an extended linear structure and, of course, dragging their substituents along with them. The three solvents studied in the simulation $-\mathrm{CHCl}_{3}, \mathrm{CHFClBr}$, and $\mathrm{H}_{3} \mathrm{C}-$ 
$\mathrm{CH}(\mathrm{OH})-\mathrm{CF}_{3}$ (called TFIPA) - each being slow to respond, at least on the timescale of the chemistry of the solute. From the rate-determining transition state to the bifurcation point in the reaction path, the solute dropped in potential energy by $\sim 40$ $\mathrm{kcal} / \mathrm{mol}$, according to the DFT calculations against which the EVB potential had been parameterized. This substantial drop in solute PE imparted considerable momentum to certain atoms in the solute, which then 'collided' with the solvent molecules in the first shell, leading to strong repulsive interactions. Crucially, these occurred before the "decision" about which enantiomer of the allene should be formed by a given trajectory (see Fig. 5).

As a consequence, when enantiomerically pure TFIPA was used in the simulation, an enantiomeric excess of $\sim 15 \%$ in the allene products was found. This is at least an order of magnitude bigger than any known optical induction caused by equilibrium solvation. The effects seen in this simulation reinforce the idea, outlined in Section 2, that solvent responses to changes in shape of the solute can be highly nonlinear. Possible consequences of this conclusion are outlined in the next Section.

\section{Future Prospects}

In the context of the themes of this Perspective, experiments that can report in real time on the dynamics of a reactive intermediate and simultaneously capture the concerted response of the solvent molecules will provide important new insights.

Perhaps advances in coherent, multidimensional ultrafast spectroscopy will deliver this level of detail. Alternatively, the expected rapid advances in femtosecond X-ray and electron diffraction, building on recent developments in sources, may soon afford direct and time-resolved images of reactive intermediates and the surrounding solvent molecules.

The topic of collision-induced IVR probably merits more attention than it has received to date. There is evidence that such a phenomenon exists, ${ }^{126}$ but the details of how and when it occurs are still unclear. The relevance to the present Perspective is that the transfer into solution of a reaction known to experience significant nonstatistical dynamical effects in the gas phase might lead to more statistical behavior if collisioninduced IVR were prevalent.

On the computational front, it appears that there is still room for improvement of solvent models. There is a real need for 'off the shelf' methods to simulate formation and decay of intermediates in solution in an accurate yet affordable way. Equilibrium solvation may well be a useful approximation for many simulations, but in the chemistry of reactive intermediates it is unlikely to be. The issue of slow response of solvents to changes in shape of a solute has received scant attention to date, but may turn out to be quite important. In particular, ongoing and still preliminary work suggests that this phenomenon can lead to the appearance of dynamical barriers to reaction, which do not necessarily occur at conventional saddle points on the PES. In general, the traditional view of solvents as relatively passive modifiers of the gas-phase PES may not turn out to be adequate, as the active participation of the medium in the overall reaction becomes clearer, both from experiment and simulation.

Finally, we note that the very definition of a reactive intermediate - the topic with which we began this Perspective - might require revision or refinement in the future as both experimental and computational methods continue to give us more detailed insights. For example, recent work has highlighted the existence of an "entropic intermediate" in a cycloaddition reaction. ${ }^{127}$ The phenomenon observed in MD simulation is of a species having significant lifetime and yet not corresponding to a local minimum on the PES. ${ }^{127}$ It occurs because of dynamical bottlenecks to product formation. In the cited example, these dynamical effects occur within the reacting molecule, but dynamical bottlenecks can occur within the solvent as well, ${ }^{128}$ raising the possibility that "entropic intermediates" might occur in solution without any counterparts in the gas phase.

\section{ACKNOWLEDGMENT}

AJOE thanks the ERC for support through Advanced Grant 290966 CAPRI.

\section{REFERENCES}

(1) Moss, R. A., Platz, M. S., Jones, Jr. M., Eds. Reactive Intermediate Chemistry; Wiley: New York, 2005.

(2) Murrell, J. N.; Laidler, K. J. Trans. Faraday Soc. 1968, 64, 371.

(3) Zuman, P.; Patel, R. Techniques in Organic Reaction Kinetics; Wiley-Interscience: New York, 1984; p. 59.

(4) Baer, T.; Hase, W. L. Unimolecular Reaction Dynamics; Oxford University Press: New York, 1996.

(5) Doering, W. von E.; Sachdev, K. J. Am. Chem. Soc. 1974, 96, 1168.

(6) Woodward, R. B.; Hoffmann, R. Angewandte Chemie-Int. Ed. 1969, $8,781$.

(7) Berson, J. A.; Nelson, G. L. J. Am. Chem. Soc. 1967, 89, 5503.

(8) Berson, J. A.; Dervan, P. B.; Malherbe, R.; Jenkins, J. A. J. Am. Chem. Soc. 1976, 98, 5937.

(9) Berson, J. A.; Malherbe, R. J. Am. Chem. Soc. 1975, 97, 5910.

(10) Baldwin, J. E. J. Comput. Chem. 1998, 19, 222.

(11) Lewis, D. K.; Brandt, B.; Crockford, L.; Glenar, D. A.; Rauscher, G.; Rodriguez, J.; Baldwin, J. E. J. Am. Chem. Soc. 1993, 115, 11728.

(12) Klarner, F. G. Topics Stereochem. 1984, 15, 1.

(13) Klarner, F. G.; Drewes, R.; Hasselmann, D. J. Am. Chem. Soc. 1988, 110, 297.

(14) Roth, W. R.; Martin, M. Tetrahedron Lett. 1967, 4695

(15) Jimenez-Oses, G.; Liu, P.; Matute, R. A.; Houk, K. N. Angewandte Chemie-Int. Ed. 2014, 53, 8664.

(16) Reyes, M. B.; Carpenter, B. K. J. Am. Chem. Soc. 2000, 122, 10163.

(17) Hrovat, D. A.; Duncan, J. A.; Borden, W. T. J. Am. Chem. Soc. 1999, 121, 169.

(18) Yamataka, H.; Sato, M.; Hasegawa, H.; Ammal, S. C. Faraday Discuss. 2010, 145, 327.

(19) Rehbein, J.; Carpenter, B. K. PCCP 2011, 13, 20906.

(20) Carpenter, B. K. Аnnu. Rev. Phys. Chem. 2005, 56, 57.

(21) Collins, P.; Carpenter, B. K.; Ezra, G. S.; Wiggins, S. J. Chem. Phys. 2013, 139, 154108.

(22) Collins, P.; Kramer, Z. C.; Carpenter, B. K.; Ezra, G. S.; Wiggins, S. J. Chem. Phys. 2014, 141, 034111.

(23) de Souza, M. A. F.; Correra, T. C.; Riveros, J. M.; Longo, R. L. J. Am. Chem. Soc. 2012, 134, 19004.

(24) Glowacki, D. R.; Marsden, S. P.; Pilling, M. J. J. Am. Chem. Soc. 2009, 131, 13896.

(25) Glowacki, D. R.; Rose, R. A.; Greaves, S. J.; Orr-Ewing, A. J.; Harvey, J. N. Nature Chem. 2011, 3, 850.

(26) Goldman, L. M.; Glowacki, D. R.; Carpenter, B. K. J. Am. Chem. Soc. 2011, 133, 5312.

(27) Kramer, Z. C.; Carpenter, B. K.; Ezra, G. S.; Wiggins, S. J. Phys. Chem. A 2015, 119, 6611.

(28) Mauguière, F. A. L.; Collins, P.; Ezra, G. S.; Farantos, S. C.; Wiggins, S. J. Chem. Phys. 2014, 140, 134112. 
(29) Merrer, D. C.; Doubleday, C. J. Phys. Org. Chem. 2011, 24, 947.

(30) Pemberton, R. P.; Hong, Y. J.; Tantillo, D. J. Pure Appl. Chem. 2013, 85, 1949.

(31) Quijano, L. M. M.; Singleton, D. A. J. Am. Chem. Soc. 2011 , $133,13824$.

(32) Samanta, D.; Rana, A.; Schmittel, M. J. Org. Chem. 2015, 80, 2174

(33) Tork, L.; Jimenez-Oses, G.; Doubleday, C.; Liu, F.; Houk, K. N. J.Am. Chem. Soc. 2015, 137, 4749.

(34) Ussing, B. R.; Hang, C.; Singleton, D. A. J. Am. Chem. Soc. 2006, 128, 7594.

(35) Yamamoto, Y.; Hasegawa, H.; Yamataka, H. J. Org. Chem. 2011, 76, 4652.

(36) Yang, Z. Y.; Doubleday, C.; Houk, K. N. J. Chem. Theory Comput. 2015, 11, 5606.

(37) Lindemann, F. A. Trans. Faraday Soc. 1922, 17, 598

(38) Parker, A. J. Chem. Rev. 1969, 69, 1

(39) Ritchie, C. D.; Skinner, G. A.; Badding, V. G. J. Am. Chem. Soc. 1967, 89, 2063.

(40) Andrews, G. D.; Baldwin, J. E. J. Am. Chem. Soc. 1977, 99, 4853.

(41) Farneth, W. E.; Damore, M. B.; Brauman, J. I. J. Am. Chem Soc. 1976, 98, 5546.

(42) Flowers, M. C.; Frey, H. M. J. Am. Chem. Soc. 1972, 94, 8636.

(43) Kevill, D. N.; D'Souza, M. J. J. Chem. Res.-S 2008, 61.

(44) Khudyakov, I.; Zharikov, A. A.; Burshtein, A. I. J. Chem. Phys. 2010, 132, 014104

(45) Kruck, M.; Munoz, M. P.; Bishop, H. L.; Frost, C. G.; Chapman, C. J.; Kociok-Kohn, G.; Butts, C. P.; Lloyd-Jones, G. C. Chem. Eur. J. 2008, 14, 7808.

(46) Bader, J. S.; Berne, B. J. J. Chem. Phys. 1996, 104, 1293.

(47) Cammi, R.; Frediani, L.; Mennucci, B.; Ruud, K. J. Chem. Phys. 2003, 119, 5818.

(48) Cramer, C. J.; Truhlar, D. G. Chem. Rev. 1999, 99, 2161.

(49) Acevedo, O.; Jorgensen, W. L. Acc. Chem. Res. 2010, 43, 142.

(50) Gao, J. L. Acc. Chem. Res. 1996, 29, 298.

(51) Aherne, D.; Tran, V.; Schwartz, B. J. J. Phys. Chem. B 2000, 104, 5382.

(52) Bernardi, E.; Martins, M. M.; Stassen, H. Chem. Phys. Lett. 2005, 407, 171.

(53) Pavlicek, N.; Schuler, B.; Collazos, S.; Moll, N.; Perez, D.; Guitian, E.; Meyer, G.; Pena, D.; Gross, L. Nature Chem. 2015, 7,623 .

(54) Becker, E. D.; Pimentel, G. C. J. Chem. Phys. 1956, 25, 224.

(55) Jacox, M. E. Chem. Soc. Rev. 2002, 31, 108.

(56) Mutunga, F. M.; Anderson, D. T. J. Phys. Chem. A 2015, 119, 2420.

(57) Hernandez, F. J.; Brice, J. T.; Leavitt, C. M.; Liang, T.; Raston, P. L.; Pino, G. A.; Douberly, G. E. J. Chem. Phys. 2015, 143, 164304.

(58) Hernandez, F. J.; Brice, J. T.; Leavitt, C. M.; Pino, G. A.; Douberly, G. E. J. Phys. Chem. A 2015, 119, 8125.

(59) Mclauchlan, K. A.; Steiner, U. E. Mol. Phys. 1991, 73, 241.

(60) Woodward, J. R. Prog. React. Kinetics Mech. 2002, 27, 165

(61) Maeda, K.; Henbest, K. B.; Cintolesi, F.; Kuprov, I.; Rodgers, C. T.; Liddell, P. A.; Gust, D.; Timmel, C. R.; Hore, P. J. Nature 2008, 453, 387.

(62) Orr-Ewing, A. J. J. Chem. Phys. 2014, 140, 090901

(63) Megerle, U.; Pugliesi, I.; Schriever, C.; Sailer, C. F.; Riedle, E. Appl Phys B-Lasers O 2009, 96, 215.

(64) Riedle, E.; Bradler, M.; Wenninger, M.; Sailer, C. F.; Pugliesi, I. Faraday Discuss. 2013, 163, 139.

(65) Greetham, G. M.; Burgos, P.; Cao, Q. A.; Clark, I. P.; Codd, P. S.; Farrow, R. C.; George, M. W.; Kogimtzis, M.; Matousek, P.; Parker, A. W.; Pollard, M. R.; Robinson, D. A.; Xin, Z. J.; Towrie, M. Appl. Spectrosc. 2010, 64, 1311.

(66) Murdock, D.; Ingle, R. A.; Sazanovich, I. V.; Clark, I. P.; Harabuchi, Y.; Taketsugu, T.; Maeda, S.; Orr-Ewing, A. J.; Ashfold, M. N. R. PCCP 2016, 18, 2629.
(67) Greaves, S. J.; Rose, R. A.; Oliver, T. A. A.; Glowacki, D. R. Ashfold, M. N. R.; Harvey, J. N.; Clark, I. P.; Greetham, G. M.; Parker, A. W.; Towrie, M.; Orr-Ewing, A. J. Science 2011, $331,1423$.

(68) Murdock, D.; Harris, S. J.; Luke, J.; Grubb, M. P.; Orr-Ewing, A. J.; Ashfold, M. N. R. PCCP 2014, 16, 21271.

(69) Rottger, K.; Marroux, H. J.; Grubb, M. P.; Coulter, P. M.; Bohnke, H.; Henderson, A. S.; Galan, M. C.; Temps, F.; OrrEwing, A. J.; Roberts, G. M. Angew. Chem. Int. Ed. 2015, 54, 14719.

(70) Grubb, M. P.; Orr-Ewing, A. J.; Ashfold, M. N. R. Rev. Sci. Instrum. 2014, 85 .

(71) Abou-Chahine, F.; Preston, T. J.; Dunning, G. T.; Orr-Ewing, A. J.; Greetham, G. M.; Clark, I. P.; Towrie, M.; Reid, S. A. J. Phys. Chem. A 2013, 117, 13388.

(72) Crowther, A. C.; Carrier, S. L.; Preston, T. J.; Crim, F. F. J. Phys. Chem. A 2008, 112, 12081.

(73) Rivera, C. A.; Winter, N.; Harper, R. V.; Benjamin, I.; Bradforth, S. E. PCCP 2011, 13, 8269 .

(74) Dunning, G. T.; Glowacki, D. R.; Preston, T. J.; Greaves, S. J.; Greetham, G. M.; Clark, I. P.; Towrie, M.; Harvey, J. N.; OrrEwing, A. J. Science 2015, 347, 530.

(75) Dunning, G. T.; Preston, T. J.; Orr-Ewing, A. J.; Greaves, S. J.; Greetham, G. M.; Clark, I. P.; Towrie, M. PCCP 2014, 16, 16095

(76) Auboeck, G.; Chergui, M. Nature Chemistry 2015, 7, 629.

(77) Roberts, G. M.; Marroux, H. J. B.; Grubb, M. P.; Ashfold, M. N. R.; Orr-Ewing, A. J. J. Phys. Chem. A 2014, 118, 11211.

(78) Middleton, C. T.; de La Harpe, K.; Su, C.; Law, Y. K.; CrespoHernandez, C. E.; Kohler, B. Annu. Rev. Phys. Chem. 2009, 60, 217.

(79) Schreier, W. J.; Gilch, P.; Zinth, W. Annu. Rev. Phys. Chem. 2015, 66, 497.

(80) Hunt, N. T. Chem. Soc. Rev. 2009, 38, 1837.

(81) Hamm, P.; Zanni, M. Concepts and Methods of 2D Infrared Spectroscopy; Cambridge University Press: Cambridge, UK, 2011

(82) Brixner, T.; Mancal, T.; Stiopkin, I. V.; Fleming, G. R. J. Chem. Phys. 2004, 121, 4221.

(83) Hybl, J. D.; Albrecht, A. W.; Faeder, S. M. G.; Jonas, D. M. Chem. Phys. Lett. 1998, 297, 307.

(84) West, B. A.; Moran, A. M. J. Phys. Chem. Lett. 2012, 3, 2575.

(85) Cahoon, J. F.; Sawyer, K. R.; Schlegel, J. P.; Harris, C. B. Science 2008, 319, 1820.

(86) Fayer, M. D. Annu. Rev. Phys. Chem. 2009, 60, 21.

(87) Hunt, N. T. Dalton Trans. 2014, 43, 17578.

(88) Oliver, T. A. A.; Fleming, G. R. J. Phys. Chem. B 2015, 119, 11428

(89) Oliver, T. A. A.; Lewis, N. H. C.; Fleming, G. R. Proc. Natl. Acad. Sci. (USA) 2014, 111, 10061.

(90) Faubel, M.; Steiner, B.; Toennies, J. P. J. Chem. Phys. 1997, 106, 9013.

(91) Winter, B.; Weber, R.; Schmidt, P. M.; Hertel, I. V.; Faubel, M.; Vrbka, L.; Jungwirth, P. J. Phys. Chem. B 2004, 108, 14558

(92) Suzuki, T. Int. Rev. Phys. Chem. 2012, 31, 265.

(93) King, S. B.; Yandell, M. A.; Neumark, D. M. Faraday Discuss. 2013, 163, 59

(94) King, S. B.; Yandell, M. A.; Stephansen, A. B.; Neumark, D. M. J. Chem. Phys. 2014, 141, 224310.

(95) Suzuki, Y. I.; Shen, H.; Tang, Y.; Kurahashi, N.; Sekiguchi, K.; Mizuno, T.; Suzuki, T. Chem. Sci. 2011, 2, 1094.

(96) Chergui, M. Faraday Discuss. 2014, 171, 11.

(97) Milne, C. J.; Penfold, T. J.; Chergui, M. Coord. Chem. Rev. 2014, 277, 44

(98) Katayama, T.; Inubushi, Y.; Obara, Y.; Sato, T.; Togashi, T.; Tono, K.; Hatsui, T.; Kameshima, T.; Bhattacharya, A.; Ogi, Y.; Kurahashi, N.; Misawa, K.; Suzuki, T.; Yabashi, M. Appl. Phys. Lett. 2013, 103

(99) Ogi, Y.; Obara, Y.; Katayama, T.; Suzuki, Y. I.; Liu, S. Y.; Bartlett, N. C. M.; Kurahashi, N.; Karashima, S.; Togashi, T.; 
Inubushi, Y.; Ogawa, K.; Owada, S.; Rubesova, M.; Yabashi, M.; Misawa, K.; Slavicek, P.; Suzuki, T. Struc. Dynamics-US 2015, 2.

(100) Silatani, M.; Lima, F. A.; Penfold, T. J.; Rittmann, J.; Reinhard, M. E.; Rittmann-Frank, H. M.; Borca, C.; Grolimund, D.; Milne, C. J.; Chergui, M. Proc. Natl. Acad. Sci. (USA) 2015, $112,12922$.

(101) Zhang, W.; Alonso-Mori, R.; Bergmann, U.; Bressler, C.; Chollet, M.; Galler, A.; Gawelda, W.; Hadt, R. G.; Hartsock, R. W.; Kroll, T.; Kjaer, K. S.; Kubicek, K.; Lemke, H. T.; Liang, H. W.; Meyer, D. A.; Nielsen, M. M.; Purser, C.; Robinson, J. S.; Solomon, E. I.; Sun, Z.; Sokaras, D.; van Driel, T. B.; Vanko, G.; Weng, T.-C.; Zhu, D.; Gaffney, K. J. Nature 2014, $509,345$.

(102) Wernet, P.; Kunnus, K.; Josefsson, I.; Rajkovic, I.; Quevedo, W.; Beye, M.; Schreck, S.; Gruebel, S.; Scholz, M.; Nordlund, D.; Zhang, W.; Hartsock, R. W.; Schlotter, W. F.; Turner, J. J.; Kennedy, B.; Hennies, F.; de Groot, F. M. F.; Gaffney, K. J.; Techert, S.; Odelius, M.; Foehlisch, A. Nature 2015, 520, 78.

(103) Attar, A. R.; Bhattacherjee, A.; Leone, S. R. J. Phys. Chem. Lett. 2015, 6, 5072.

(104) Miller, R. J. D. Annu. Rev. Phys. Chem. 2014, 65, 583.

(105) Manz, S.; Casandruc, A.; Zhang, D.; Zhong, Y.; Loch, R. A.; Marx, A.; Hasegawa, T.; Liu, L. C.; Bayesteh, S.; DelsimHashemi, H.; Hoffmann, M.; Felber, M.; Hachmann, M.; Mayet, F.; Hirscht, J.; Keskin, S.; Hada, M.; Epp, S. W.; Floettmann, K.; Miller, R. J. D. Faraday Discuss. 2015, 177, 467.

(106) Bagchi, B.; Jana, B. Chem. Soc. Rev. 2010, 39, 1936.

(107) Jimenez, R.; Fleming, G. R.; Kumar, P. V.; Maroncelli, M. Nature 1994, 369, 471.

(108) Rosenthal, S. J.; Jimenez, R.; Fleming, G. R.; Kumar, P. V.; Maroncelli, M. J. Mol. Liq. 1994, 60, 25.

(109) Funkner, S.; Niehues, G.; Schmidt, D. A.; Heyden, M.; Schwaab, G.; Callahan, K. M.; Tobias, D. J.; Havenith, M. J. Am. Chem. Soc. 2012, 134, 1030.

(110) Xu, Y.; Havenith, M. J. Chem. Phys. 2015, 143, 170901.
(111) Abou-Chahine, F.; Greaves, S. J.; Dunning, G. T.; Orr-Ewing, A. J.; Greetham, G. M.; Clark, I. P.; Towrie, M. Chem. Sci. 2013, 4, 226.

(112) Glowacki, D. R.; Orr-Ewing, A. J.; Harvey, J. N. J. Chem. Phys. 2015, 143, 044120.

(113) Glowacki, D. R.; Orr-Ewing, A. J.; Harvey, J. N. J. Chem. Phys. 2011, 134, 214508.

(114) Rose, R. A.; Greaves, S. J.; Abou-Chahine, F.; Glowacki, D. R.; Oliver, T. A. A.; Ashfold, M. N. R.; Clark, I. P.; Greetham, G. M.; Towrie, M.; Orr-Ewing, A. J. PCCP 2012, 14, 10424.

(115) Calaway, W. F.; Ewing, G. E. Chem. Phys. Lett. 1975, 30, 485.

(116) Litovitz, T. A. J Acoust Soc Am 1956, 28, 770.

(117) Oyola, Y.; Singleton, D. A. J. Am. Chem. Soc. 2009, 131, 3130.

(118) Glowacki, D. R.; Liang, C. H.; Marsden, S. P.; Harvey, J. N.; Pilling, M. J. J. Am. Chem. Soc. 2010, 132, 13621.

(119) Ufimtsev, I. S.; Martinez, T. J. Comput Sci Eng 2008, 10, 26.

(120) Vreven, T.; Morokuma, K. J. Phys. Chem. A 2002, 106, 6167.

(121) Warshel, A.; Weiss, R. M. J. Am. Chem. Soc. 1980, 102, 6218.

(122) Chang, Y. T.; Miller, W. H. J. Phys. Chem. 1990, 94, 5884.

(123) Carpenter, B. K. Chem. Rev. 2013, 113, 7265.

(124) Ussing, B. R.; Singleton, D. A. J. Am. Chem. Soc. 2005, 127, 2888.

(125) Carpenter, B. K.; Harvey, J. N.; Glowacki, D. R. PCCP 2015, $17,8372$.

(126) Weston, R. E., Jr.; Barker, J. R. J. Phys. Chem. A, 2006, 110, 7888.

(127) Patel, A.; Chen, Z.; Yang, Z.; Gutiérrez, O.; Liu, H.; Houk, K. N.; Singleton, D. A. J. Am. Chem. Soc. 2016, ASAP, DOI: 10.1021/jacs.6b00017.

(128) Dhaliwal, M.; Basilevsky, M. V.; Weinberg, N. J. Chem. Phys. 2007, 126, 234505 . 